\title{
COMMENT
}

\section{Unifying metastasis - integrating intravasation, circulation and end-organ colonization}

Jacob Scott ${ }^{1,2}$, Peter Kuhn ${ }^{3}$ and Alexander R. A. Anderson ${ }^{1}$

Abstract | Recent technological advances that have enabled the measurement of circulating tumour cells (CTCs) in patients have spurred interest in the circulatory phase of metastasis. Techniques that do not solely rely on a blood sample allow substantial biological interrogation beyond simply counting CTCs.

In patients with advanced primary cancer, circulating tumour cells (CTCs) ${ }^{1}$ can be found throughout the entire vascular system ${ }^{2}$. When and where these CTCs form metastasis is not fully understood, and is currently the subject of intensive biological study. Paget's well-known seed-soil hypothesis ${ }^{3}$ suggests that the 'soil' (the site of a metastasis) is as important as the 'seed' (the metastatic cells) in the determination of successful metastasis. The mechanism by which seeds are disseminated to specific soil has, to date, been a 'known unknown'. We think that it is during this poorly understood phase of metastasis that we stand to answer important questions ${ }^{4}$.

We hypothesize that the rich variety of possible metastatic disease patterns not only stems from the physical aspects of the circulation but also from CTC heterogeneity (FIG. 1). These seeds represent many different populations that are derived from a diverse population of competing phenotypes within the primary tumour ${ }^{5}$. Because such seeds need to pass through a system of physical and biological filters in the form of specific organs, the circulatory phase of metastasis could be modelled as a complex deterministic filter. In theory, until the evolution of a suitable seed, any number of CTCs could flow through the circulation and arrest at end organs without metastases forming. As tumour heterogeneity is thought to expand as the tumour progresses, it follows that at some point a seed will come into existence that is suited to a specific soil within that patient's body. If this seed is to propagate it must find its soil, a process that we hypothesize is governed by solvable physical rules that relate to the dynamics of the circulatory flow between different organs and how these organs filter (not only by size, but probably also by other biological mechanisms). Although these biological mechanisms are not yet known, we might be able to infer their existence by finding out which measurements do not fit a model that is defined only by physical flow and filtration.

To begin the process of physical interrogation, we propose a model that represents the human circulatory system as a directed and weighted network, with nodes representing organs and edges representing arteries and veins. The novelty is only fully realized when combined with a heterogeneous CTC population (driven by primary tumour heterogeneity) modulated by the complex organ filter system (with physiologically relevant connections) under dynamic flow. Four important biological processes emerge from this representation. First, the shedding rate, which is defined as the rate at which the tumour sheds CTCs into the vasculature. Second, CTC heterogeneity, which is defined as the distribution of CTC phenotypes present in the circulation. Third, the filtration fraction, which is defined as the proportion (and type) of CTCs that arrest in a given organ. Fourth, the clearance rate, which is defined as the rate at which cancer cells are cleared from the blood and/or organ after arrest. Each of these biological processes is probably disease- and even patient-specific, and each is extremely poorly understood.

Using this representation to motivate the development of a mathematical model we can define both the concentration of CTCs and their phenotypic distribution at any given point in the network, as well as organspecific filtration values. To parameterize this model, characterization and enumeration of CTCs taken from a single patient at different time points and from different points in this network will need to be undertaken. A complete understanding of the model will also provide information about the behaviour of the system as a whole. Specifically, the average lifespan of a CTC in a patient's circulation will be able to be calculated with only a minimum of measurements. Although this seems to be a simple calculation, the scientific literature on this 


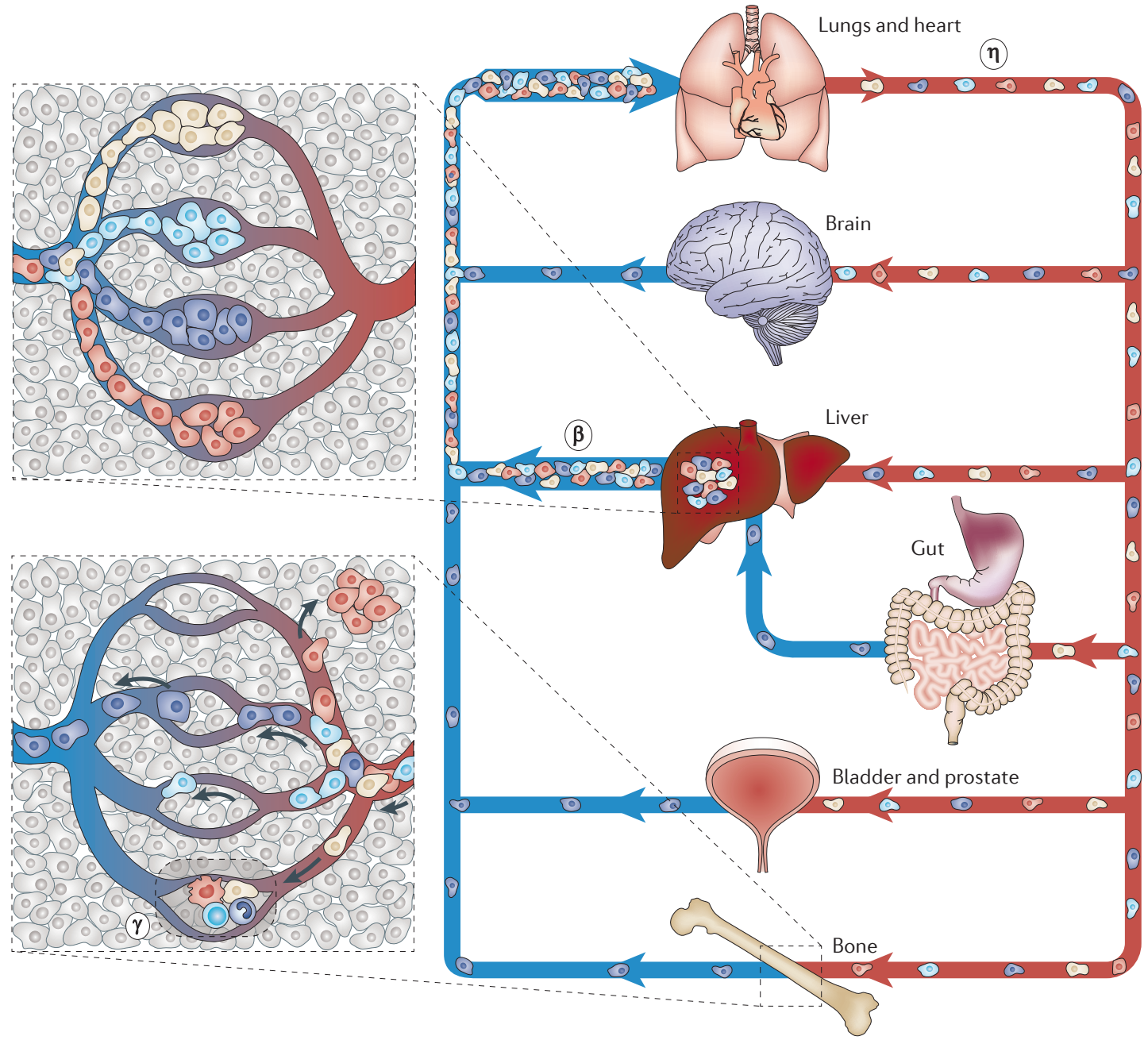

Figure 1| A simplified representation of the human vascular system connecting representative organs with a primary tumour in the liver. Four phenotypes representing putatively different metastatic clones are represented by the four different colours. The shedding term, $\beta$, represents the rate and type of cells exiting the primary tumour. The clearance rate, $\gamma$, represents the rate at which cells are removed from the system by an immune response or other processes. The filtration fraction, $\eta$, represents the proportion of cells that arrest in an organ per pass and can be thought of as the concentration of cells coming out of an organ divided by the concentration going into the organ. In this example, the red cells are the proper 'seed' for the 'soil' of the bone. It is easy to see in this case how a red cell would have difficulty finding the bone, as it could be arrested in the lung or sent out to other organs and arrested there. The dark blue cells, which are able to flow through capillary beds, represent a circulator phenotype.

topic is substantially conflicted, with reports of cells lasting in the order of years in some patients ${ }^{6}$ but less than 24 hours $^{7}$ in others. It seems likely that even this simple factor will differ between patients and cancer types and will lead to many questions: could there be a 'circulator' phenotype that avoids arrest with extreme efficiency that can explain long dormancy periods? Could this cell type be targeted? Could such a cell type be mechanically filtered out? This example represents one of the many new pieces of information that we are on the brink of understanding through mathematical models that could inform treatment decision-making paradigms.

\footnotetext{
Marrinucci, D. et al. Case study of the morphologic variation of circulating tumor cells. Hum. Pathol. 38, 514-519 (2007).

2. Jiao, L. R. et al. Unique localization of circulating tumor cells in patients with hepatic metastases. J. Clin. Oncol. 27, 6160-6165 (2009).

3. Paget, S. The distribution of secondary growths in cancer of the breast. 1889. Cancer Metastasis Rev. 8, 98-101 (1989).
}

4. Valastyan, S. \& Weinberg, R. Tumor metastasis: molecular insights and evolving paradigms. Cell 147, 275-292 (2011).

5. Gerlinger, M. et al. Intratumor heterogeneity and branched evolution revealed by multiregion sequencing. N. Engl. J. Med. 366 883-892 (2012).

6. Meng, S. et al. Circulating tumor cells in patients with breast cancer dormancy. Clin. Cancer Res. 10, 8152-8162 (2004).

7. Stott, S. L. et al. Isolation and characterization of circulating tumor cells from patients with localized and metastatic prostate cancer. Science Transl Med. 2, 25ra23 (2010).

\section{Acknowledgements}

The authors would like to thank K. Kadyshevskaya for her help with the figure. This work was supported by PS-OC grant numbers U54CA 143970 and U54CA143906 from the National Cancer Institute (NCI), USA. The content is solely the responsibility of the authors and does not necessarily represent the views of the $\mathrm{NCl}$.

Competing interests statement

The authors declare no competing financial interests.

\section{FURTHER INFORMATION}

Alexander R. A. Anderson's homepage: http://labpages.moffitt.org/andersona/ ALL LINKS ARE ACTIVE IN THE ONLINE PDF 